\title{
Gift-Me : Personalized Gift Recommender System
}

\author{
Taware Shruti S. ${ }^{1}$, Yadav Krushna M $^{1 *}$, Khatri Pavan D. ${ }^{1 *}$
}

\begin{abstract}
Nowadays most of the india market is moving towards an online world with an adroit to have an maximum market scope. So we are connecting the business of gift article to online world with the evolved versions of current algorithm of recommendation systems.Ecommerce is an online site where the sale or purchase of goods are ordered electronically. The available ecommerce system have some issues with the recommendation so we are collaborating the multiple algorithm to increase the product sale and convinient in user interaction.
\end{abstract}

Keywords - Collaborative Filtering,Content based recommendation,Hybrid recommendation,Knowledge mining

\section{INTRODUCTION}

$\mathrm{R}$ ecommender system apply statical and knowledge discovery technique to the problem of product recommender during live customer intereaction and they are acheiving wide spread in sucess of ecommerce. In our project we apply several technique of analising large scale purchase and preference data for the purpose producing use ful recommendation to the customer in particular we apply collection of algorithm and traditional datamining , collaborative filtering, knowledge mining and sentimental analysis. We device different tecnique for diff sub-processes and apply their combination over dataset to compare for recommendation quality and performance. The focus of this project is two fold :

- we provide systematic evaluation of diffrent technique for recommender system and

- Represent new algorithm that are particular suited for sparse dataset such as those that are comman in.

\section{Problem Statement:}

In this system we are working on the theme of Gift Shop actually we wanted to design the gift recommendation system including other facilities. It aims to alert people of available of various personalised gift item to increase his sale and to suggest gift according to his need or by user behavioral analysis .

In this proposed system we face two challenges together by studying new and existing algorithm that have the potential that improve both scalability and quality of recommendation system. Their has been little work on experiemental validation of recommendor system against a set of real world database.More experiemental validation against real world data set and it is important that this dataset include ecommerce data as well content data.

${ }^{1}$ Department of Computer Engineering,

SVPM's COE Malegoan (Bk), Savitribai Phule Pune University, Maharashtra.

Let, set Users ' $U^{\prime}=\{\mathrm{U} 1, \mathrm{U} 2, \mathrm{U} 3, \ldots ., \mathrm{Un}\}$ interact with the system 'S'with dataset 'D' of parameter,such as select productP1,P2,P3...,Pn, users interest product are represented as a product Matrix ' $M '=\{R 1, R 2, R 3 \ldots ., R n\}$. Each user can rate a product $\mathrm{Pu}=\{\mathrm{Pj} 1, \mathrm{Pj} 2, \mathrm{Pj} 3 \ldots \mathrm{Pjn}\}$, and other attribute such as User profile,likes, dislikes,past history and more information about user.At the end data will be process with certain algorithm to get intersted product as an output.

\section{CASE STUDY}

Trust and satisfaction are the main determinant for successful business relationships in business to consumer electronic commerce. Trust plays vital role in any form of businesses that require monetary transaction.Customer satisfaction has a direct relationship to customers purchase intention, repurchase intention and word of mouth marketing.consumer satisfaction is a major of how well the product or services provide by the company's meeting its consumer expections.

1.Recommendation system in E-commerce websites: A Graph BasedApproached.

\section{Advantages:-}

This paper is regarding the lack of semantic factor in recommendation systems and describes the different recommendation techniques that are being employed in the current e-commerce website. Recommendation system can be broadly classified into three categories: content-based, collaborative, and hybrid recommendation approaches.[1]

Disadvantages:-

They reviewed various limitation of the current recommendation method and have concluded that integration of semantic in recomendation technique can provide better recommendation. The proposed system is able to succesfully integrate recommendation and semantic.[1]

2.Improving Collaborative Filtering by Selecting an Effective User Neighborhood for Recommender Systems.

Advantages:-In this paper they have stated an improvised collabrative filtering by using effective user neighbor .The KNN used for get the most favourable recommendation [2] .

Disadvantages:- In this they are not using flexible technique with collaborative filtering and implementing hybrid approach which further improves the accuracy of recommendation presented to the customer. [2] 


\section{Recommender Systems in E-Commerce}

Advantages:- Create customizable product and services , provide point od delivery customization provide quick responce for value chain.[3]

Disadvantages:They are not provided creativity that is needed to produce the recommender system in ecommerce.[3]

\section{TABLE I. (ANALYSIS REPORT FOR VARIOUS ECOMMERCE WEBSITE)}

\begin{tabular}{|l|l|l|}
\hline Business & Interface & Technology \\
\hline Amazon.com & $\begin{array}{l}\text { similar } \\
\text { items } \\
\text { Email }\end{array}$ & $\begin{array}{l}\text { I2I corelation } \\
\text { Attribute } \\
\text { based } \\
\text { Attribute } \\
\text { based } \\
\text { Aggregated } \\
\text { Ratings } \\
\text { Top n list } \\
\text { Feedback Profile }\end{array}$ \\
\hline $\begin{array}{l}\text { Average } \\
\text { Ratings } \\
\text { Text } \\
\text { Comments }\end{array}$ & $\begin{array}{l}\text { Average } \\
\text { Ratings } \\
\text { Text } \\
\text { Comments }\end{array}$ & $\begin{array}{l}\text { Aggregated } \\
\text { Ratings } \\
\text { Likers }\end{array}$ \\
\hline eBay & $\begin{array}{l}\text { similar } \\
\text { items } \\
\text { Top N List }\end{array}$ & $\begin{array}{l}\text { I2I corelation } \\
\text { P2P } \\
\text { corelation }\end{array}$ \\
\hline
\end{tabular}

\section{PROPOSED SYSTEM}

Following are modules designed for this system:

- Resource Module:

- It includes various categories of gift and differen items available to them .

-They have 500+ product which they manufacturer. Eg: Co-orporate gifts , birthday gifts, gifts for him/her ,friendship day gift etc.

-Database creation for these products and categorize them. Input:Output:-

Rough information, photographs.

Database entry for products wih its attribute.
- User Module:

-System will provide registration form for login and profile creation. -User can buy gifts.

-User can give rating to that gift.

Input:-

Uses information, like, rating, history, reviews. Output:-

Data for UBA or recommendation system on that basis we ecommend gift product to user.

- Role Defining Mechanism:

-In this module we can find out trusted user. -User are categorized into three structures -Visitor, -Customer, -Admin ( Superuser).

-Authorization and Authentication happens here.

Input:-

Login details.

Output:categorized user customer / visitor,admin.

- Photo Competition:

-This module is basically include photographer world with shop.

-In this a competition will be conducted online to attract other photographer to the shop.

- Winner will be chosen through ratings and likes.

Input:-

Photos from photographer and info.

Output:competition rating and result of competition.

- Gift-Me :

-This module is helpful foor those who are little busy in there life schedule and work and cannot visit shop for gifts and this system is mainly designed for it only . - On this visitor can buy a gift and place order for their loved ones.

-Our system accept order of that gift and also at a same time customer details with receipt address .

Input:-

User information,Destination Address.

Output:-

Recommended gift.

- Portfolio Campaign:

-In this we are going to visit different business man and collect their reviews to enhance business scope a little bit and try to convince them .

-This will empower our skill and all of this activity will be digitally represented using videos and photos .

- Magical Gift: 
- In this the user will get certain exciting offer depending on transaction with client.

- It also include social media discount.

-Offers will be categorized using various reasons : Whether he/she is continuous customer, or he/she has stoped visiting website depending on certain criteria oreward / offer will be assigned

Input:-

Customer history of purchase, social media feedback. Output:-

Valid to grab offer or not if valid then assign specific offer in which he/she fits.

- Mailing Module:

- Mail server creation

- Confirmation mails , offer mails, status update are given by this module.

Input:customer details, collected from user module , ordered gift price and date.

Output:mails sent to user.

- Business Module:

-This module deals with recommendation system of website.

- Various algorithm are required to be implemented in this section for predicting user behavioral:

Hybrid Filtering, Knowledge mining, Content Based Recommendation Algorithm.

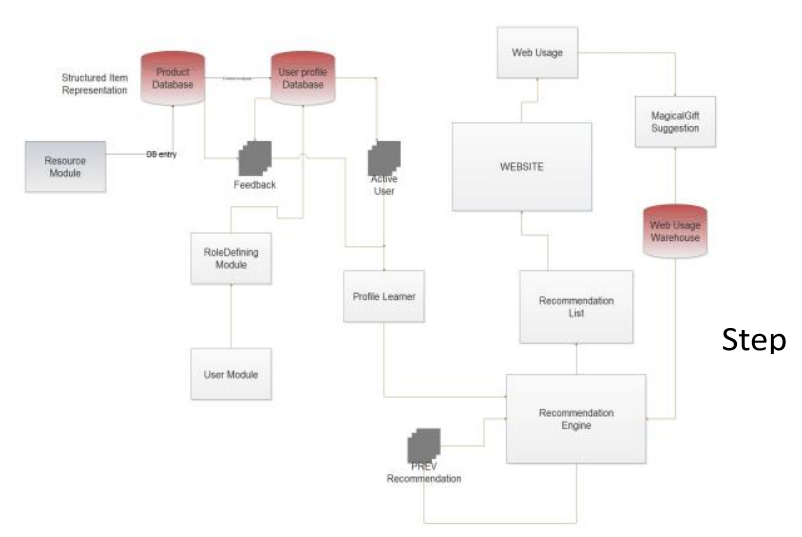

System Architecture

A. Algorithms

1) Item-to-Item based Jaccard Similarity :-

In any commerce setting, the concept of item similarity is very not straightforward. Two users usually buying same kinds of products can be considered as similar, but we cannot say the same about two items bought by same user.

Jaccard Similarity of Sets :

The Jaccard similarity of sets $\mathrm{S}$ and $\mathrm{T}$,

$\operatorname{SIM}(\mathrm{S}, \mathrm{T})=|S \cap T||S \cup T|$

Example : we see two sets $\mathrm{S}$ and $\mathrm{T}$. There are three elements in their intersection and a total of eight elements that appear in $\mathrm{S}$ or $\mathrm{T}$ or both. Thus, $\operatorname{SIM}(\mathrm{S}, \mathrm{T})=3 / 8$.

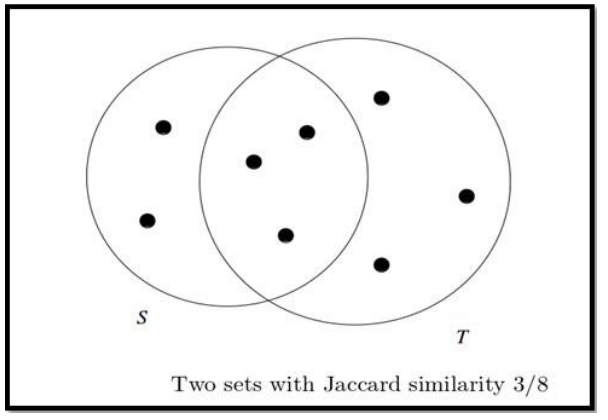

There are two different concepts of item similarity for recommendation purposes. One is, if the two items are physically similar, for example : Blue coloured key-chains and Red coloured key-chains, and other is if they have functional dependency on each other, for example: Simple printed mugs and Personalized photo mugs. For finding this similar items, one can create a dictionary of attributes defining the product and do Jaccard similarity on those attributes.

Algorithmic Steps :

Step 1 : Consider items A and B with attributes$\{u a, u b, u c, u d\}$ ie, products purchased by particular set of users

Step 2 : Let item $\mathrm{A}=\{u a, u b, u c\}$ and item $\mathrm{B}=\{u b, u c, u d\}$

3 : Similar users between items $\mathrm{A}$ and $\mathrm{B}$, $(\mathrm{A} \cap B)=\{u b, u c\}$ therefore, $\mathrm{n}(\mathrm{A} \cap$ $\mathrm{B})=2$, and Total users, $(\mathrm{A} \cup B)$ $=\{u a, u b, u c, u d\} \quad$ therefore, $\mathrm{n}(\mathrm{AU}$ B) $=4$.

Step 4 : Jaccard's Similarity is given by, $\operatorname{Jsim}(\mathrm{A}, \mathrm{B})=|A \cap B| \backslash A \cup B \mid$, where $|A \cap B| !=$ 0

Input :

Item $\mathrm{A}=\{$ color $:$ Red, size $:$ mini, material $:$ Wooden, product $:$ SimplePrintedMugs $\}$ 
Item $\mathrm{B}=\{$ color $:$ Red, size $:$ mini,material $:$ Acrylic, product $:$ PersonalizedPhotoMugs

Thus, the intersection of sets would be number of attributes that match up, i.e.

Intersection $(\mathrm{A}, \mathrm{B})=\{$ color, size $\}$

Union $(\mathrm{A}, \mathrm{B})=\{$ color, size, material, product $\}$ Jaccard Index $=$ $2 / 4=0.50$ Output predictions (filtering) about the interests of a user by collecting preferences or taste information from many

\begin{tabular}{|l|rrrrr|}
\hline Jaccard Index & Item A & Item B & Item C & Item D \\
Item A & 0.67 & 1 & 0.75 & 1 \\
Item B & 0.82 & 1 & 0.37 & 1 \\
Item C & 1 & 0.58 & 1 & 0.77 \\
Item D & 0.66 & 1 & 0.89 & 1 \\
\hline
\end{tabular}

Jaccard Index $=\{0.67,1,1,0.75,1,0.82,1,0.37,1,1,0.58,1$, $0.77,0.66,1,0.89,1\}$

Higher value of Jaccard Index, maximum is the probable time the item to be recommended.

\section{2) Collaborative Filtering(User-to-User based) :-}

Collaborative filtering is a method of making automatic users

(collaborating).

In a collaborative filtering approach, when the user asks for recommendations, the recommender first produces a list containing a specific number of users who have similar interests to the active user. The users attributes similarity is calculated by computing addition values. Maximum value indicated the recommendation of the product.

Algorithm :

Given an user $\mathrm{u} 1$, find the most similar users with their related attributes,

set bestscore $=$ minValue $(0)$

for each other user $u 2$ set matchscore $=0$ for each

feature

compare $\mathrm{u} 1$ and $\mathrm{u} 2$ on that feature if they match, increment matchscore if matchscore

$>$ bestscore bestscore $=\mathrm{u} 2$ and

bestscore $=$ matchscore

return bestmatch Example:

Input

Users $=\{$ Age, Profession, CategoryofItem $\}$

\begin{tabular}{|l|l|l|l|l|l|}
\hline $\begin{array}{l}\text { User-to-user based } \\
\text { CF }\end{array}$ & User A & User B & User C & User D & User E \\
\hline Age & 20 & 18 & 63 & 20 & 17 \\
\hline Profession & Cs Engg & $\begin{array}{l}\text { High-School } \\
\text { Student }\end{array}$ & Senior Citizen & Cs Engg & $\begin{array}{l}\text { High- } \\
\text { School } \\
\text { Student }\end{array}$ \\
\hline $\begin{array}{l}\text { Category } \\
\text { of item }\end{array}$ & $\begin{array}{l}\text { Personalized } \\
\text { Mugs }\end{array}$ & $\begin{array}{l}\text { Wooden } \\
\text { keychains }\end{array}$ & Album & $\begin{array}{l}\text { Personalized } \\
\text { Mugs }\end{array}$ & Album \\
\hline
\end{tabular}

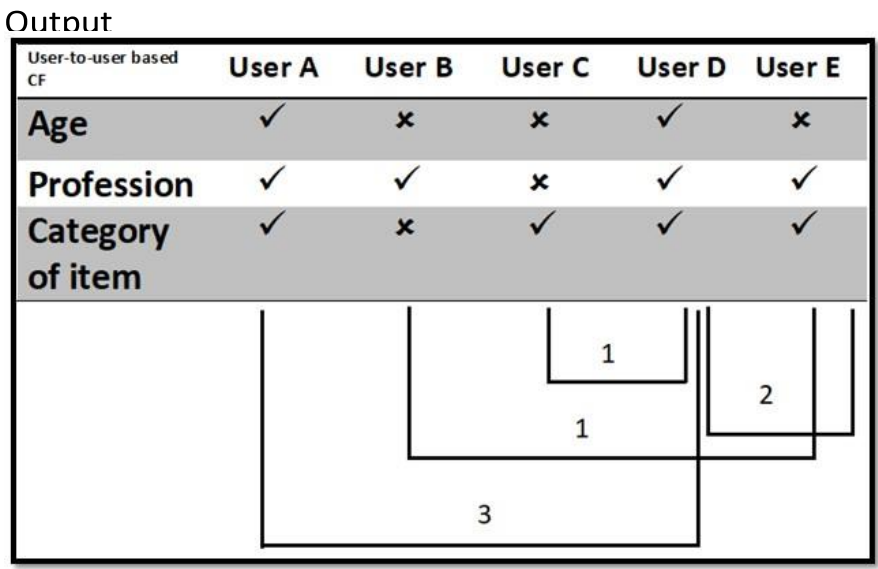

3) Content Based Recommendation Algorithm(Neighborhood based algorithm) :-

The content-based filtering approach identifies the similarity between a user and the new items using the content of the previously evaluated items in the user profile. In addition, each item in a user profile is characterized by a set of attributes which is constructed by extracting a set of features from an item. Such a profile is used to determine if the new item is similar to the item that a user has preferred in the past.

It basically works on the users' preference that is likes and dislikes given by any user to items or products and user profile,outputs of this algorithm will be a set of products.

User-to-Item based MBR Algorithm : (Input) Item profile:

Metadata ,toys.

Eg: [different personalised product, giftcards, etc]

Let dataset $\mathrm{D}$ with product $\mathrm{p} \mathrm{D}$

$=\{p 1, p 2, p 3, \ldots, p n\}$

User profile:

Let $\mathrm{U}$ be the set of user

$\mathrm{U}=\{u 1, u 2, u 3, \ldots, u n\}$

Attributes $=\{$ likes, dislikes, ratings, pastHistory, reviews $\}$ 
Output $\mathrm{S}=\{U, D, M\}$

$\mathrm{U} \times \mathrm{D} \rightarrow \mathrm{M}$

$\mathrm{M}$ : Matrix with predicted ratings $\mathrm{R}(\mathrm{i}, \mathrm{j})$

A] Initialization Stage

The first vector : num - stores information about how many users have rated an item.

The second vector : sum - stores the total rating score of the items.

$$
\mathrm{r}_{i}={\frac{\operatorname{sum}_{i}}{\text { num }_{i}}}
$$

B] M-Distance

$$
\mathrm{r}_{i}={\frac{j=0}{\text { num }_{i}}=\frac{n-1}{\left|\left\{0 \leq j \leq n-1 \mid r_{i, j} \geq 1\right\}\right|}}^{n r i, j \text { sum }_{i}}
$$

\begin{tabular}{|c|c|c|c|c|}
\hline & P0 & P1 & P2 & P3 \\
\hline U0 & 0 & 2 & - & 4 \\
\hline U1 & 4 & 0 & 3 & 0 \\
\hline U2 & 3 & 5 & 4 & 0 \\
\hline U3 & 0 & 3 & 0 & 3 \\
\hline Num(N & 2 & 3 & 2 & 2 \\
\hline Sum(S) & 7 & 10 & 7 & 7 \\
\hline$\dot{\mathbf{R}}=\mathbf{S} / \mathbf{N}$ & 3.5 & 3.3 & 3.5 & 3.5 \\
\hline
\end{tabular}

M-Distance :

$m d_{i, j}=\left|r_{i}-r_{j}\right| \leq \delta$ (threshold)

C] The MBR Algorithm

Input : $\{$ sumVector, numVector $\}=\left\{\right.$ sum $_{i}$, num $\left._{i}\right\}$

Output : Predicted ratings $\mathrm{PR}_{i, j}$

$P R i, j=$ $B(u i, p j) r i, j$

Ratings of the side neighbours of the item whose unknown ratings to be derived is given by, $\mathrm{B}\left(\mathrm{u}_{i}, p_{j}\right)$

STEP 1: Initialization:

$\mathrm{N}=[2,3,2,2]$

$\mathrm{S}=[7,10,7,7]$

STEP 2: Finding M-distance:

$\bar{R}=[3.5,3.3,3.5,3.5]$

Average ratings given by neighbour user has the range $[3.2,3.6]$

STEP 3: The MBR Algorithm:

$\mathrm{B}(\mathrm{U} 0, \mathrm{P} 2)=\{P 1, P 2\}$ Since, $\mathrm{M}(\mathrm{U} 0, \mathrm{P} 0)=0=\{P 0, P 3\}$ are not neighbour

$\operatorname{PR}(0,2)=(2+4) / 2=3$

In the updated stage the predicted rating is added in vector $\bar{R}$ Example:

\section{B. Mathematical Model \\ Let, where, \\ $\mathrm{HS}=$ sytemain work \\ $\mathrm{I}=$ Input bigdata \\ $\mathrm{S}=\mathrm{User}$ session $=\{\mathrm{S} 1, \mathrm{~S} 2, \ldots . \mathrm{Sn}\}$ Sclus $=$ list \\ of semantic cluster} $\mathrm{HS}=\{\mathrm{I}, \mathrm{S}, \mathrm{Sclus}, \mathrm{Robj}, \mathrm{F}$ CBpred,F KBPred,Pd,Uf,pr,Uin,Up,O $\}$

list $<$ Si $>=$ Bigdata entry.for $;$ product, Attribute , intrest,HobbiesViews, ProductHistory,Like/Dislike >

F CBpred=Content Based Recommendation Uf and Up F CBpred.(Sclus $=\langle U f\rangle$, Sclus $=\langle U p\rangle)$

F KBPred $=($ Sclus $=\langle U f\rangle$, Sclus $=\langle U$ in $>$. Sclus $=\langle P d\rangle)$

QSuq Pred.(input dataset of product)

$\mathrm{Pd}=$ product with its key features or description

Uf=user Profile

$\mathrm{Pr}=$ product ratings

Uin=User attribute and area of intrest $\mathrm{Up}=\mathrm{User}$ preference

Robj=Recommended product

$\mathrm{O}=$ Output Recommended list

$\mathrm{R} 1=\mathrm{F}$ CBPred

$\mathrm{R} 2=\mathrm{F}$ KBPred $\mathrm{R}=\sum(R 1+R 2)$

$\mathrm{R} 3=\mathrm{QSuq}$ pred return

$\mathrm{R}$ and $\mathrm{R} 3$

\section{RESULT}

Our result shows that dimensionality reduction technique hold the promise of allowing CF-based algorithm to scale to large dataset and at the same time produce high quality recommendation. Increase costumer aware of varoius products available in Gift items which was are main moto for designing the system . 
- Provided solutions for online marketing.

- Provided real-time guidance.

- Providied single cost services.

\section{CONCLUSION}

In this system the user is provided with an e-commerce website to buy gifts online,good shopping experience, cart designing accompanied with user friendly interaction. This is convenient for user to see product of their interest.In this proposed system we provide number of features that are designed to make the customer more comfortable. The system can make different recommendations for different users. It includes totally two user groups, new users and old users. Because we do not know new users preferences, we recommend the item which has the highest demand. Meanwhile we take full account of the old users preference to make personalized recommendations. So we can provide the best solutions for the users.

\section{FUTURE WORK}

In future we are combining various technologies with this implementation such that machine learning and IoT for automated Gift ordering system.Future work is required to understand exactly why low dimensional representation works well for some recommender application and less well for others.

\section{REFERENCES}

[1] Recommendation system in E-commerce websites: A Graph Based Approached.

Ms. Shakila Shaikh Dr. Sheetal Rathi,Asst Prof. Prachi Janrao(2017 IEEE).

[2] Improving Collaborative Filtering by Selecting an Effective User Neighborhood for Recommender Systems. Mr.Sundus Ayyaz, Usman Qamar(2017 IEEE)

[3] Recommender Systems in E-Commerce J. Ben Schafer, Joseph Konstan,John Riedl(2017 IEEE).

Department of computer science and Engineering, University of Minnesota

[4] E-commerce Recommendation method based on collaborative filltering technology.

Vipul Narayan,R.K.Mehta,Mahendra Rai,Ashish Gupta,Swati Verma Department of computer science and Engineering,Faizabad India (IEEE june 2017)

[5] Recommendation system for grocery store considering data sparsity

Natsuki sano,Natsumi Machino,Katsutoshi Yada,Tomomichi Suzuki Tokyo

University of science ,Japan(IEEE 2015) 